Rev. Elev. Méd. Vét, Poys trop., 1966, 19, 2 (137-148).

\title{
Note d'herpétologie tchadienne. Étude préliminaire de quelques serpents récoltés dans la région de Fort-Lamy de 1954 à 1965
}

\author{
par M. GRABER
}

\begin{abstract}
RÉSUMÉ
460 serpents ont été récoltés de 1954 à 1765 dans la partıe Nord de la République du Tchad, appartenant à 19 genres et à 24 espèces différentes.

L'auteur étudie particulièrement la régıon voisine du Laboratolre de Farcha (Fort-Lamy) où dominent : Psammophis sibilans sibilans (54 p. 100), Cousus rhombealus (18 p. 100), Atractaspis watsonn (9 p. 100), Naja hoje hoje (5 p. 100), Nojo nigricollis (4 p. 100), Booedon fuliginosum (4 p. 100) et Bitis arietons arletons (1 p. 100).

Dans cette zone, les serpents sont rares de novembre à mai. Ils sont beaucoup plus nombreux durant l'hivernage et en arrière-saison (octobre). Chez l'homme, le pourcentage d'envenimations ophidiennes relevé en 1956-1957 se sifue autour de 0,75 p. 100.

Chez les animaux, les piquores de serpents causent relativement peu d'accidents morrels, sauf chez les poulets.
\end{abstract}

\section{INTRODUCTION}

Les serpents du Tchad sont encore très mal connus et le territoire de la République n'a été, jusqu'à maintenant, qu'incidemment prospecté.

Dans le rapport de la mission Titho 1906-1909, Pellegrin (1914) signale l'existence de certaines espèces : Typhlops punctotus, Python sebae, Crotaphopeltis hotombera, Bitis arietans, Cerastes cerostes, Echis carinatus et Atractospis nigra. Ces espèces ont été recueillies surtout aux confins Nigero-Tchadiens.

Plus récemment, Sœur Roussel et A. Villiers (1965) donnent une première liste d'Ophidiens de la région de Gounou-Gaya (Département du Mayo-Kebbi). Depuis, 1954, la Section de Parasitologie du Laboratoire de Farcha, dans le but d'étudier les Helminthes de reptiles, a procédé à un certain nombre de sondages en divers points du Territoire.
L'enquête était suffisamment avancée, il nous a paru intéressant de publier les résultats obtenus, étant bien entendu que la liste dont it sera question ici n'est que provisoire et qu'elle devra être complétée ultérieurement.

\section{MATÉRIEL — LIEUX DE CAPTURE}

460 serpents au total ont été examinés de 1954 à 1965 :

$$
\begin{array}{r}
32 \text { en } 1954 \\
50 \text { en } 1955 \\
60 \text { en } 1956 \\
286 \text { en } 1957 \\
9 \text { en } 1959 \\
6 \text { en } 1961 \\
2 \text { en } 1963 \\
15 \text { en } 1965
\end{array}
$$


Retour au menu

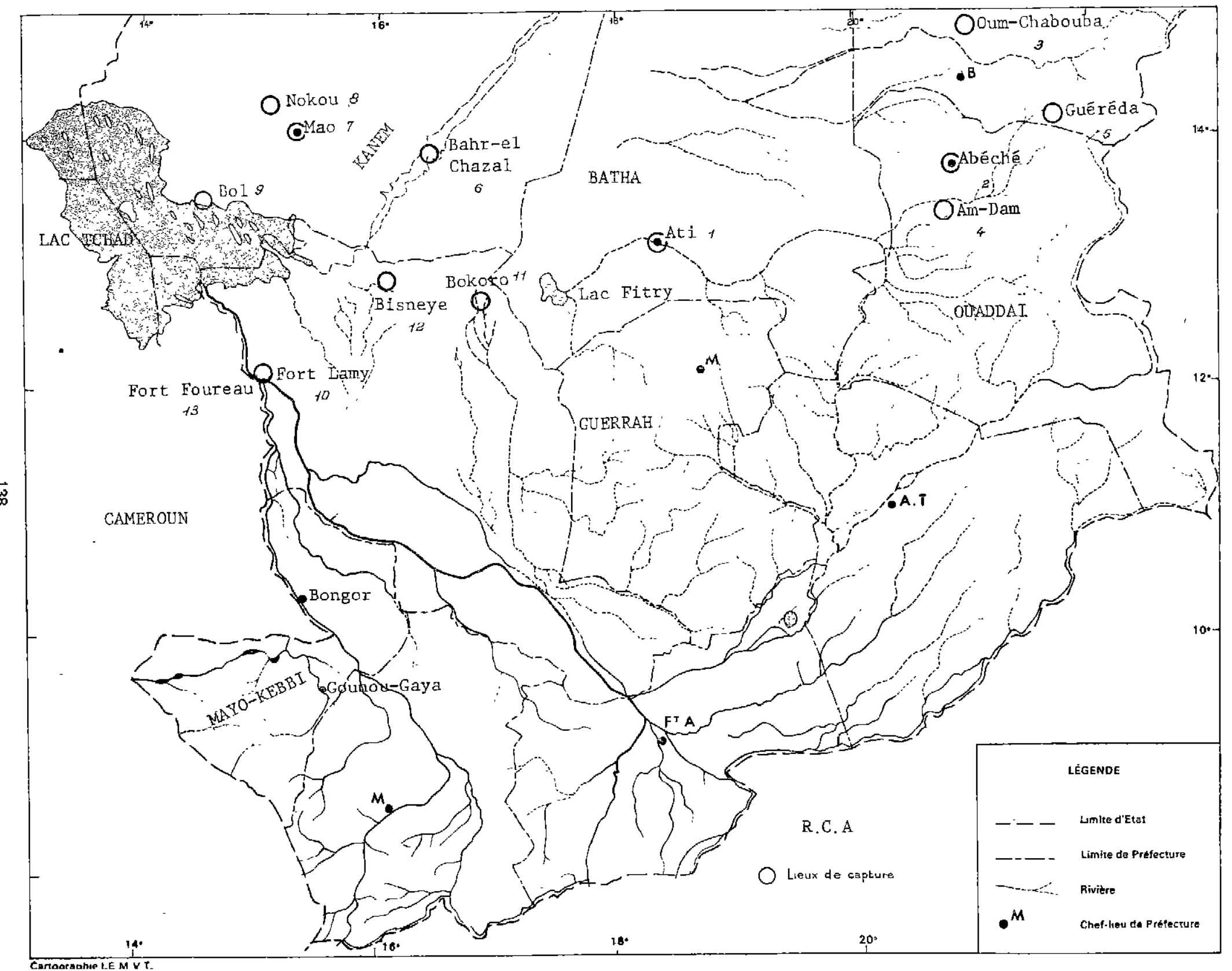


Ils étalent originarres des régions sulvantes (voir carte No 1).

Batha : 4 (Ati, point No 1)

Ouaddai : 14 (Abécher, 2 ; Oum-Chalouba, 3 ; Am-Dam, 4 ; Guéreda, 5).

Kanem : 7 (Bahr-el-Ghazal, 6; Mao, 7, Nokou, $8 ; \mathrm{Bol}, 9)$.

Chart-Baguirmi : 430 (Fort-Lamy, 10; Bokoro, 11 ; Bisneye, 12).

Nord-Cameroun : 2 (Fort-Foureau, 13).

Ennedi : 3.

D'une façon générale, la zone étudiée s'étend du $12^{\mathrm{e}}$ au $16^{\mathrm{e}}$ parallèle, de l'isohyète 600 à l'isohyète 200 : elle couvre donc deux types de clımat (Sahélo-soudanien et Sahélo-saharien).

En réalité, seule la sous-préfecture de FortLamy a fait l'objet d'un inventaire assez complet, portant sur plus de 400 spécimens. Le Laboratoire a servi et sert encore bénévolement de centre de traitement pour les personnes du voisınage piquées par des serpents. Il est alors facile de collecter le matériel qui vient parfois d'assez loin à la ronde (plus de 20 kilomètres). C'est dire que la zone plate et marécageuse située à f'Ouest du confluent Logone-Charı a été très largement inventorlée.

\section{LISTE DES ESPÈCES RENCONTREEES}

\section{I. - Famille des Typhlopidae.}

Typhlops punctatus Leach.

Nombre : 4

Origine : Bisneye, Fort-Lamy, également MayoKebbi (Roussel et Villiers, 1965).

\section{II. - Famille des Boidae.}

10 Python seboe Gmelin

Nombre : 8

Origine : Bol, Nokou ; Fort-Lamy ; Ouaddaï ; Bokoro; Batha, également Mayo-Kebbi (Roussel ef Villiers, 1965).

\section{$2^{\circ}$ Eryx muelleri mueller Boulenger}

Nombre: 1

Origine : Bahr-el-Ghazal, également MayoKebbi (Roussel et Villiers, 1965).

\section{Eryx colubrina Linné}

Nombre : 5 Mao.
III. - Famille des Colubridae.

10 Bocedon fuliginosum fuliginosum Baié

Nombre: 13

Origine : Fort-Lamy, également Mayo-Kebbi (Roussel et Villiers, 1965).

A l'origine, un grand nombre d'espèces ont été incorporées dans le genre Bocedon. Roux Estève ef Guibé (1965) comparant B. fuliginosum (Boié) et $B$. lineatum (Duméril et Bibron) arrivent à la conclusion que ces deux espèces africaines ne peuvent être différenciées et que $B$. lineatum doit être mis en synonymie avec $B$. fuliginosum.

Sur les 13 spécimens examinés à Fort-Lamy. six d'entre eux pouvaient être rattachés à la forme Lineatum et les sept autres à la forme Fuliginosum.

Mensurations ef écaillure : voir tableau no 1 ,

20 Philothomnus irregularis irregularis Leach

Nombre: 4

Origine : Fort-Lamy, également Mayo-Kebbi (Roussel et Villiers, 1965).

$3^{\circ}$ Meizodon coronotus Schlege!.

Nombre : 5

Origine: Fort-Lamy, Abécher, également Mayo-Kebbi (Roussel ef Villiers, 1965).

4o Prosymna meleagris Reinhardt.

Nombre : 4

Origine : Abécher ; Fort-Lamy, également Mayo-Kebbi (Roussel et Villiers, 1965).

50 Scophiophis albopunctatus albopunctatus Peters

Origine :' Fort-Lamy, également Mayo-Kebbi (Roussel et Villiers, 1965).

Nombre : 1

$6^{\circ}$ Dasypeltis scobra scabra Linné

Nombre: 2

Origine : Fort-Lamy, également Mayo-Kebbı (Roussel et Viliters, 1965).

7o Crotaphopeltis hotamboeia Laurentı

Nombre : 5

Origine : Fort-Lamy ; Ałi ; Bisneye, également Mayo-Kebbı (Roussel et Villiers, 1965).

80 Romphiophis oxyrhynchus Reinhardt

Nombre : 3

Origine : Fort-Lamy, également Mayo-Kebbi (Roussel et Villiers, 1965). 
TABLEAU No I

Boaedon fultginosum - mensurations et écaillure.

\begin{tabular}{|c|c|c|c|c|c|c|c|c|c|}
\hline \multirow[b]{2}{*}{$\begin{array}{l}\text { Exemplaire } \\
\text { numéro }\end{array}$} & \multicolumn{2}{|c|}{ Mensurations } & \multicolumn{7}{|c|}{ Ecaillure } \\
\hline & $\begin{array}{l}\text { Longueur } \\
\text { totale }\end{array}$ & $\begin{array}{l}\text { Longuevir } \\
\text { de la } \\
\text { queue }\end{array}$ & Rangs & Ventrales & $\begin{array}{c}\text { Sous } \\
\text { caudales }\end{array}$ & $\begin{array}{c}\text { Labiales } \\
\text { supérieures }\end{array}$ & Préoculaires & Postoculaires & Temporales \\
\hline $\begin{array}{l}1 \\
2 \\
3 \\
4 \\
5 \\
6 \\
7\end{array}$ & $\begin{array}{l}768 \mathrm{~mm} \\
750 \mathrm{~mm} \\
280 \mathrm{~mm} \\
795 \mathrm{~mm} \\
500 \mathrm{~mm} \\
483 \mathrm{~mm} \\
850 \mathrm{~mm}\end{array}$ & $\begin{array}{l}110 \mathrm{~mm} \\
97 \mathrm{~mm} \\
40 \mathrm{~mm} \\
95 \mathrm{~mm} \\
95 \mathrm{~mm} \\
85 \mathrm{~mm} \\
95 \mathrm{~mm}\end{array}$ & $\begin{array}{l}27 \\
27 \\
29 \\
29 \\
27 \\
31 \\
31\end{array}$ & $\begin{array}{l}231 \\
228 \\
209 \\
233 \\
217 \\
232 \\
236\end{array}$ & $\begin{array}{l}48 \\
50 \\
50 \\
53 \\
65 \\
65 \\
49\end{array}$ & $\begin{array}{l}8 \\
8 \\
8 \\
8 \\
8 \\
8 \\
8\end{array}$ & $\begin{array}{l}1 \\
1 \\
1 \\
2 \\
1 \\
1 \\
2\end{array}$ & $\begin{array}{l}2 \\
2 \\
2 \\
2 \\
2 \\
2 \\
2\end{array}$ & $\begin{array}{l}1+2 \\
1+2 \\
1+2 \\
1+2 \\
1+3 \\
1+2 \\
1+4\end{array}$ \\
\hline
\end{tabular}

TABLEAU No II

Psammophis gibilans - mensurations et écaillure.

点

\begin{tabular}{|c|c|c|c|c|c|c|c|c|c|}
\hline \multirow[b]{2}{*}{$\begin{array}{l}\text { Exemplaire } \\
\text { numér }\end{array}$} & \multicolumn{2}{|c|}{ Mensurations } & \multicolumn{7}{|c|}{ Ecaillure } \\
\hline & $\begin{array}{l}\text { Longueur } \\
\text { totele }\end{array}$ & $\begin{array}{l}\text { Longueus } \\
\text { de la } \\
\text { queue }\end{array}$ & Range & Ventrales & $\begin{array}{c}\text { Sous } \\
\text { caudales }\end{array}$ & $\begin{array}{l}\text { Labiales } \\
\text { supérieures }\end{array}$ & Préoculaires & Postoculaires & Temporales \\
\hline $\begin{array}{r}1 \\
2 \\
3 \\
4 \\
5 \\
6 \\
7 \\
8 \\
9 \\
10 \\
11 \\
12 \\
13 \\
14 \\
15 \\
16 \\
17 \\
18 \\
19\end{array}$ & $\begin{array}{r}1.186 \mathrm{~mm} \\
1.040 \mathrm{~mm} \\
877 \mathrm{~mm} \\
1.100 \mathrm{~mm} \\
1.260 \mathrm{~mm} \\
895 \mathrm{~mm} \\
510 \mathrm{~mm} \\
385 \mathrm{~mm} \\
730 \mathrm{~mm} \\
443 \mathrm{~mm} \\
1.267 \mathrm{~mm} \\
380 \mathrm{~mm} \\
632 \mathrm{~mm} \\
650 \mathrm{~mm} \\
1.500 \mathrm{~mm} \\
740 \mathrm{~mm} \\
770 \mathrm{~mm} \\
892 \mathrm{~mm} \\
1.270 \mathrm{~mm}\end{array}$ & $\begin{array}{c}344 \mathrm{~mm} \\
300 \mathrm{~mm} \\
222 \mathrm{~mm} \\
270 \mathrm{~mm} \\
266 \mathrm{~mm} \\
260 \mathrm{~mm} \\
\text { mutilée } \\
119 \mathrm{~mm} \\
220 \mathrm{~mm} \\
119 \mathrm{~mm} \\
373 \mathrm{~mm} \\
115 \mathrm{~mm} \\
184 \mathrm{~mm} \\
200 \mathrm{~mm} \\
370 \mathrm{~mm} \\
210 \mathrm{~mm} \\
203 \mathrm{~mm} \\
255 \mathrm{~mm} \\
310 \mathrm{~mm}\end{array}$ & $\begin{array}{l}17 \\
17 \\
17 \\
17 \\
17 \\
17 \\
17 \\
17 \\
17 \\
17 \\
17 \\
17 \\
17 \\
17 \\
17 \\
17 \\
17 \\
17 \\
17\end{array}$ & $\begin{array}{l}172 \\
168 \\
176 \\
175 \\
169 \\
172 \\
165 \\
161 \\
170 \\
174 \\
176 \\
165 \\
174 \\
171 \\
174 \\
165 \\
172 \\
165 \\
166\end{array}$ & $\begin{array}{r}102 \\
100 \\
82 \\
97 \\
102 \\
92 \\
- \\
90 \\
98 \\
95 \\
97 \\
107 \\
103 \\
91 \\
81 \\
77 \\
95 \\
93 \\
73\end{array}$ & $\begin{array}{l}8 \\
8 \\
8 \\
8 \\
8 \\
8 \\
8 \\
8 \\
8 \\
8 \\
8 \\
8 \\
8 \\
8 \\
8 \\
8 \\
8 \\
8 \\
8\end{array}$ & $\begin{array}{l}1 \\
1 \\
1 \\
1 \\
1 \\
1 \\
1 \\
2 \\
2 \\
1 \\
1 \\
1 \\
1 \\
2 \\
1 \\
1 \\
1 \\
2 \\
1\end{array}$ & $\begin{array}{l}2 \\
2 \\
2 \\
2 \\
2 \\
2 \\
2 \\
2 \\
2 \\
2 \\
2 \\
2 \\
2 \\
2 \\
2 \\
2 \\
2 \\
2 \\
2 \\
2 \\
2 \\
2 \\
2\end{array}$ & $\begin{array}{l}2+3 \\
2+3 \\
2+3 \\
2+2 \\
2+2 \\
2+2 \\
2+3 \\
2+3 \\
2+3 \\
2+2 \\
2+3 \\
2+3 \\
2+3 \\
2+3 \\
2+3 \\
2+3 \\
2+3 \\
2+3 \\
2+3\end{array}$ \\
\hline
\end{tabular}


90 Dromophis lineatus Duméril et Bibron.

Nombre : 1

Origine : Fort-Lamys également Mayo-Kebbi (Rousel et Vilitiers, 1965).

100 Psammophis schokari Forskal

Nombre : 3

Origine: Abécher; Fort-Lamy; Bahr-el-Ghazal. Mensurations ef écaillure (un exémplaire)

Mensurations :

Ecaillure

Longueur : $1,55 \mathrm{~m}$

Longueur de la queve : $41 \mathrm{~cm}$

Nombre de rangs : 17

Nombre de ventrales : 192

Nombre de sous-caudales : 118

Nombre de labiales supérieures: 9

Nombre de temporales : $2+2$,

110 Psammophis sibilans sibılans Lınné

Nombre : 228

Origine: Fort-Lamy ; Oum-Chalouba ; Kokoro, également Mayo-Kebbi (Roussel et Villiers, 1965).

Cette couleuvre se présente sous deux formes:

- l'une unicolore, brun olivâtre.

- l'autre à tête brun clair, dos brun foncé avec deux larges bandes latérales et une ligne médiane parfois interrompue.

Ces deux variantes sont très classiques dans toute l'Afrique au Sud du Sahara.

Mensuratıons et écaillure (voir tableau $n^{\circ} 2$ ).

\section{IV. - Famille des Elapidae.}

10 Noja haje haje Linné

Nombre : 22
Origine : Fort-Lamy; Abécher, également Mayo-Kebbi (Roussel et Villiers, 1965).

Mensurations et écaillure (voir tableau no $\mathrm{lll}$ ).

$2^{\circ}$ Noja melonoleuca Hallowell.

Un exemplaire recueıllı par Mr. le Dr vétérinaire Latour à Fort-Archambault, en 1965.

30 Naja nigricollis Reinhardt

Nombre : 19

Origine : Fort-Lamy ; Fort-Foureau ; Abécher, également Mayo-Kebbi (Roussel et Villiers, 1965).

Mensurations et écaillure (voir tableau no IV).

\section{V. - Famille des Viperidae.}

10 Causus rhombeotus Lichtenstein

Nombre : 78

Origine : Fort-Lamy ; Bokoro ; Abécher, également Mayo-Kebbi (Roussel et Villiers, 1965).

Mensurations et écaillure (voir tableau $n^{\circ} \mathrm{V}$ ).

$2^{\circ}$ Bits arietons arietans Merrem

Nombre: 9

Origine : Fort-Lamy ; Fort-Foureau ; Abécher; Ati, également Mayo-Kebbi (Roussel et Villiers, 1965).

\section{3o Echis carinatus Schneider}

\section{Nombre : 7}

Origine: Abécher et Ouaddai; Bahr-el-Ghazal ; Bisneye, également Mayo-Kebbı (Roussel et Villiers, 1965).

Cette vipère est extrêmement fréquente dans l'Est du Tchad (Ovaddaï ; Ennedi ; Zagahoua) où elle provoque des accidents mortels chaque année. Elle existe en abondance au Bahr-elGhazal. Elle semble beaucoup plus rare au

TABLEAU Ho III

Naja haje - mensurations et écatllure

\begin{tabular}{|c|c|c|c|c|c|c|c|}
\hline \multirow[b]{2}{*}{$\begin{array}{l}\text { Eremplaire } \\
\text { nunéro }\end{array}$} & \multicolumn{2}{|c|}{ Kensuretiong } & \multicolumn{5}{|c|}{ Ecaillure } \\
\hline & $\begin{array}{l}\text { Longueur } \\
\text { totale }\end{array}$ & $\begin{array}{l}\text { Longueur } \\
\text { de la } \\
\text { queue }\end{array}$ & Rengs & Ventrales & $\begin{array}{c}\text { Sous } \\
\text { caudales }\end{array}$ & Pér1sculaires & Pemporales \\
\hline$=1$ & $1.385 \mathrm{mtn}$ & $210 \mathrm{~mm}$ & 21 & 215 & 53 & 6 & $1+3$ \\
\hline 2 & $480 \mathrm{~mm}$ & $80 \mathrm{~mm}$ & 21 & 210 & 64 & 6 & $1+3$ \\
\hline 3 & $1.675 \mathrm{~mm}$ & $220 \mathrm{~mm}$ & 21 & 213 & 50 & 6 & $1+3$ \\
\hline 4 & $1.500 \mathrm{IIII}$ & $300 \mathrm{cms}$ & 21 & 207 & 51 & 6 & $1+3$ \\
\hline
\end{tabular}


TABLEAD NO IV

Naja niericollis - mengurations et écaillure.

\begin{tabular}{|c|c|c|c|c|c|c|c|c|c|}
\hline \multirow[b]{2}{*}{$\begin{array}{l}\text { Exemplairo } \\
\text { numéro }\end{array}$} & \multicolumn{2}{|c|}{ Mensuraticns } & \multicolumn{7}{|c|}{ Ecaillure } \\
\hline & $\begin{array}{l}\text { Longueur } \\
\text { totale }\end{array}$ & $\begin{array}{l}\text { Longueur } \\
\text { de la } \\
\text { queue }\end{array}$ & Rangs & Ventrales & $\begin{array}{c}\text { Sous } \\
\text { caudales }\end{array}$ & $\begin{array}{l}\text { Irabiales } \\
\text { supérieures }\end{array}$ & Préoculaires & Postoculaires & Temporales \\
\hline 1 & $580 \mathrm{~mm}$ & $104 \mathrm{~mm}$ & 22 & 197 & 62 & 6 & 2 & 3 & $2+5$ \\
\hline 2 & $966 \mathrm{~mm}$ & $175 \mathrm{~mm}$ & 21 & 194 & 58 & 6 & 2 & 3 & $2+5$ \\
\hline 3 & $1.410 \mathrm{~mm}$ & 250 m & 21 & 195 & 60 & 6 & 2 & 3 & $2+4$ \\
\hline 4 & $1.500 \mathrm{~mm}$ & $2.50 \mathrm{mall}$ & 22 & 199 & 55 & 6 & 2 & 3 & $2+4$ \\
\hline
\end{tabular}

TABLEAU NO $\mathrm{V}$

$\stackrel{\leftrightarrow}{\mathbb{N}}$

Causus rhombeatus - mensurations et écaillure.

\begin{tabular}{|c|c|c|c|c|c|c|c|c|c|c|}
\hline \multirow[b]{2}{*}{$\begin{array}{l}\text { Exemplaife } \\
\text { numéro }\end{array}$} & \multicolumn{2}{|c|}{ Mensurations } & \multicolumn{8}{|c|}{ Ecailluce } \\
\hline & $\begin{array}{l}\text { Longueur } \\
\text { totale }\end{array}$ & $\begin{array}{l}\text { Longueur } \\
\text { de la } \\
\text { queue }\end{array}$ & Rargs & Ventrales & $\begin{array}{l}\text { Sous } \\
\text { caudales }\end{array}$ & $\begin{array}{l}\text { tabiales } \\
\text { supérıcurres }\end{array}$ & Préoculaires & Postoculaireg & $\begin{array}{l}\text { Sous } \\
\text { oculaires }\end{array}$ & Temporales \\
\hline $\begin{array}{r}1 \\
2 \\
3 \\
4 \\
5 \\
6 \\
7 \\
8 \\
9 \\
10 \\
11 \\
12 \\
13 \\
14 \\
15 \\
16\end{array}$ & $\begin{array}{l}465 \mathrm{~mm} \\
550 \mathrm{~mm} \\
596 \mathrm{~mm} \\
430 \mathrm{~mm} \\
442 \mathrm{~mm} \\
460 \mathrm{~mm} \\
520 \mathrm{~mm} \\
540 \mathrm{~mm} \\
573 \mathrm{~mm} \\
422 \mathrm{~mm} \\
587 \mathrm{~mm} \\
535 \mathrm{~mm} \\
535 \mathrm{~mm} \\
515 \mathrm{~mm} \\
530 \mathrm{~mm} \\
562 \mathrm{~mm}\end{array}$ & $\begin{array}{l}37 \mathrm{~mm} \\
50 \mathrm{~mm} \\
57 \mathrm{~mm} \\
43 \mathrm{~mm} \\
45 \mathrm{~mm} \\
50 \mathrm{~mm} \\
50 \mathrm{~mm} \\
45 \mathrm{~mm} \\
47 \mathrm{~mm} \\
43 \mathrm{~mm} \\
47 \mathrm{~mm} \\
50 \mathrm{~mm} \\
48 \mathrm{~mm} \\
53 \mathrm{~mm} \\
40 \mathrm{~mm} \\
55 \mathrm{~mm}\end{array}$ & $\begin{array}{l}20 \\
19 \\
18 \\
17 \\
19 \\
19 \\
19 \\
19 \\
19 \\
19 \\
19 \\
19 \\
19 \\
20 \\
19 \\
19\end{array}$ & $\begin{array}{l}149 \\
150 \\
150 \\
148 \\
148 \\
126 \\
145 \\
153 \\
153 \\
153 \\
143 \\
153 \\
157 \\
149 \\
151 \\
148\end{array}$ & $\begin{array}{l}22 \\
25 \\
24 \\
26 \\
25 \\
20 \\
22 \\
21 \\
23 \\
27 \\
20 \\
27 \\
20 \\
26 \\
19 \\
24\end{array}$ & $\begin{array}{l}6 \\
6 \\
6 \\
6 \\
6 \\
6 \\
6 \\
6 \\
6 \\
6 \\
6 \\
6 \\
6 \\
6 \\
6 \\
6\end{array}$ & $\begin{array}{l}2 \\
2 \\
2 \\
2 \\
2 \\
2 \\
2 \\
2 \\
2 \\
2 \\
2 \\
2 \\
2 \\
2 \\
2 \\
2 \\
2 \\
2\end{array}$ & $\begin{array}{l}2 \\
2 \\
2 \\
2 \\
2 \\
2 \\
2 \\
2 \\
2 \\
2 \\
2 \\
2 \\
2 \\
2 \\
2 \\
2 \\
2\end{array}$ & $\begin{array}{l}2 \\
2 \\
2 \\
2 \\
2 \\
2 \\
1 \\
2 \\
2 \\
2 \\
2 \\
2 \\
2 \\
2 \\
2 \\
2 \\
2 \\
2\end{array}$ & $\begin{array}{l}2+3 \\
2+3 \\
2+3 \\
2+3 \\
2+3 \\
2+3 \\
2+3 \\
2+3 \\
2+3 \\
2+3 \\
2+3 \\
2+3 \\
2+3 \\
2+3 \\
2+3 \\
2+3\end{array}$ \\
\hline
\end{tabular}


Chari-Baguirmı : dans la région de Fort-Lamy, elle est, jusqu'à plus ample informé, rarissime.

40 Cerostes cerostes Linné

Nombre : 2

Origine : Ennedi et Bahr-el-Ghazal.

$5^{\circ}$ Cerastes vipero Linné

Nombre : 1

Origine: Tibesti.

Ces deux serpents sont des espèces sahariennes ou sub-sahariennes.

\section{Atractaspis watsonii Boulenger}

Nombre : 39

Origine : Fort-Lamy.

Suivant la latitude, on rencontre au Tchad des peuplements divers :

- de type soudano-guinéen comme Cousus rhombeatus, Philothomnus irregularis, Naja nigricollis ;

- de type soudanien comme Booedon fuliginosum et Prosymmo meleagridis ;

- de type sahélo-saharien comme Eryx colubrino, Psammophis schokori ou Naja haje :

- de type saharien comme Cerastes cerostes.

Dans la région de Fort-Lamy, sauf en ce qui concerne cette dernière espèce, ces peuplements sont pratiquement confondus.

\section{IMPORTANCE RELATIVE DES ESPÈCES}

Parmı les serpents récoltés à Fort-Lamy, les espèces les plus communes sont dans l'ordre décroissant (sur 413 reptiles) :

Psammophis sibilans sibilans : 54 p. 100

Cousus rhombeatus : 18 p. 100

Atractaspis watsonu: 9 p. 100

Noja haje haje: 5 p. 100

Naja nigricollis: 4 p. 100

Booedon fuliginosum : 4 p. 100

Bitıs orietans arietans : 1 p. 100

Colubridés divers : 5 p. 100.

L'importance relative des espèces dans la zone de Fort-Lamy est donc différente de celle donnée par Roussel et Villiers pour Gounou-Gaya (MayoKebbi) où dominent sur environ 200 exemplaires) :

Echis carinatus : 15 p. 100

Causus rhombeotus : 15 p. 100
Cousus resimus : 15 p. 100

Psommophis sibilans : 10 p. 100

\section{RÉPARTITION DANS LE TEMPS DES PRINCIPALES ESPÈCES}

\section{A. - Répartition globale annuelle.}

Les observations ont été effectuées d'octobre 1956 à décembre 1957 sur une aire de 10 kilomètres $\times 10$ kilomètres ayant pour sommet le Laboratoire de Farcha ef bordée sur toute sa longueur par le fleuve Chari.

308 serpents ramassés sur ce périmètre ont été amenés au Laboratoire selon le rythme suivant :

Octobre $1956: 7,8$ p. 100

Novembre $1956: 4,5$ p. 100

Décembre $1956: 1,7$ p. 100

Janvier $1957: 4,8$ p. 100

Février $1957: 2,9$ p. 100

Mars $1957: 4,2$ p. 100

Avril $1957: 4,8$ p. 100

Mai $1957: 2,2$ p. 100

Juin $1957: 8,8$ p. 100

Juillet $1957: 12,4$ p. 100

Aout $1957: 9,7$ p. 100

Septembre $1957: 6,3$ p. 100

Octobre $1957: 25,7$ p. 100

Novembre $1957: 4,2$ p. 100 .

D'une façon générale, de novembre à mai, les serpents sont relativement rares. Cette période correspond à la saison sèche. lis ne réapparaissent qu'au début de la saison des pluies. Durant I'hivernage (juin à octobre), ils sont assez nombreux, le moss d'octobre étant le plus favorable à leur mise en évidence, du fait des débroussements liés à des travaux agricoles.

\section{B. - Répartition mensuelle par espèce.}

Les graphiques I et II donnent les répartitions mensuelles pour les cinq espèces les plus importantes. Leur lecture indique :

10 Psammophis sibilans se trouve constamment présent, les récoltes les plus fournies ayant lieu en octobre.

$2^{\circ}$ Les Noja-haje et nigricollis se voient surtout de février à novembre avec un «trou »en mai.

3o Booedon fuliginosum est un serpent d'hivernage. 
GRAPHIQUE $n^{\circ}$ I Répartition mensuelle par especes

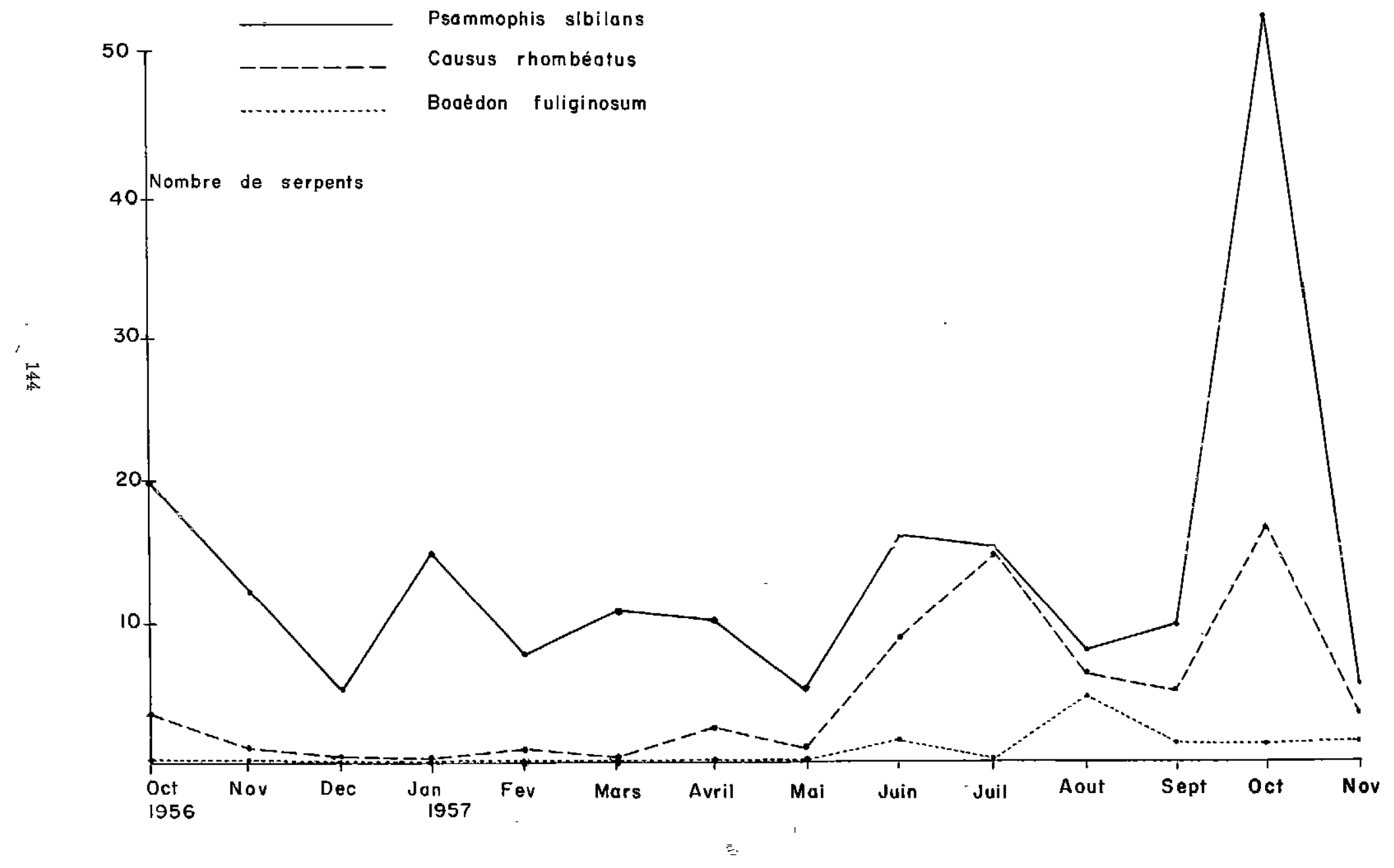




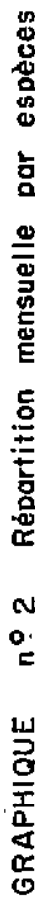

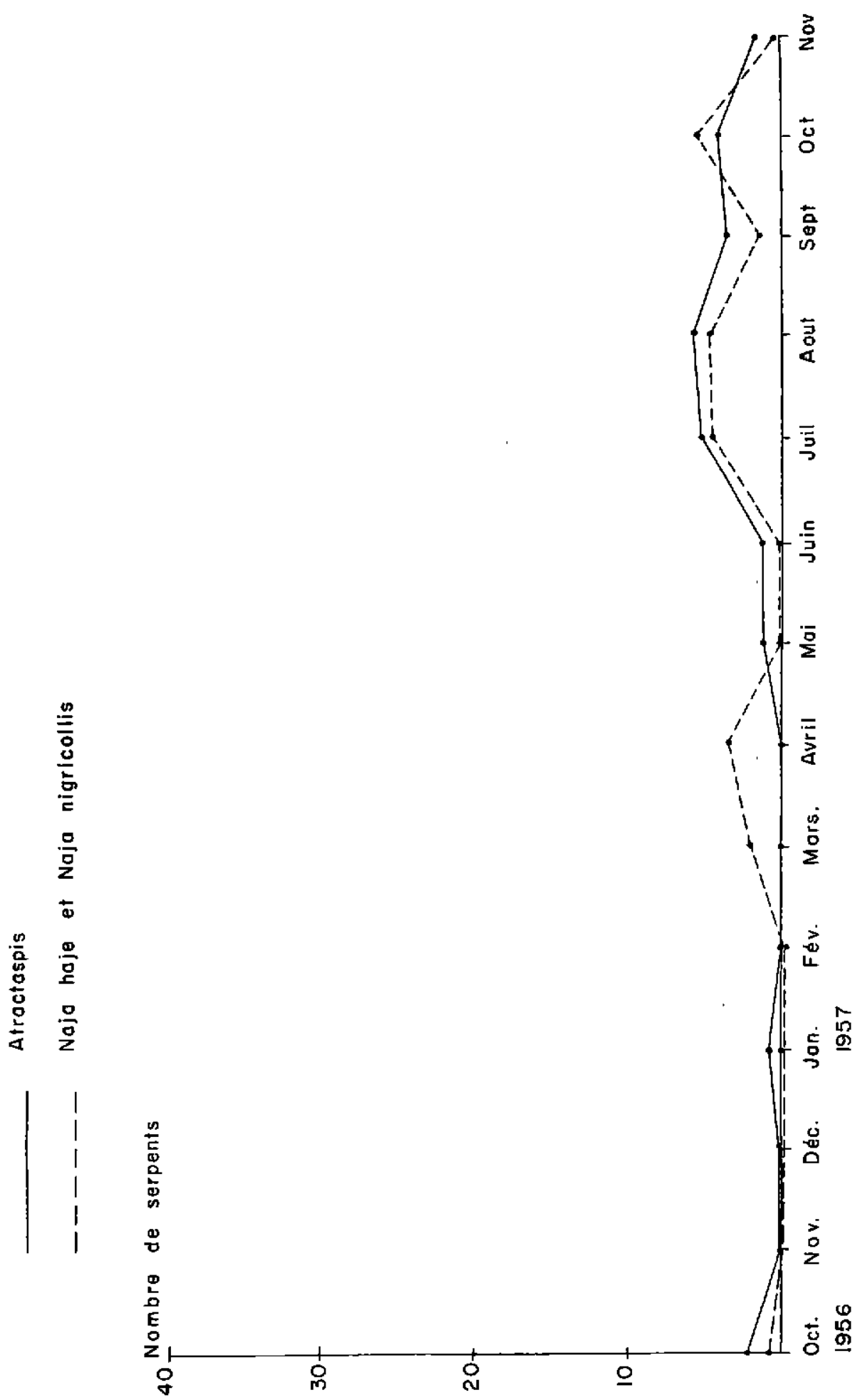


40 II en est de même pour Causus rhombeatus et Atractaspis qui abondent de mars-avril jusqu'en novembre. Ils sembient disparaître à peu près totalement par la suite.

\section{ACCIDENTS CAUSÉS PAR LES SERPENTS}

II ne sera question ici que des observations faltes dans la zone étudiée.

a) Chez l'homme, en 1956-57, sur une population globale évaluée à 2.000 habitants, 15 personnes ont été soignées pour piqûres de serpents, ce qui représente 0,75 p. 100. Les accidents sont surtout le fait des Causus et des Atractaspis, vipères qui fréquentent volontiers les lieux d'habitation ef les anfractuosités des murs. Leur morsure se traduit par un malaise général pouvantaller jusqu'à la syncope, malaise qui s'atténue peu à peu et par des réactions locales violentes : gonflement et hémorragies au voisinage du point d'inoculation avec parfois comme conséquence de la nécrose ef de l'atrophie.

Ont été notés également des accidents oculaires (conjonctivites) par projection de venin de Najo nigricollis et quelques morsures de Bitis arietans qui ont été jugulées grâce à l'admınistration quasi immédiate du sérum correspondant.

b) Chez les animaux, sur plus de 7.000 ruminants utilisés au Laboratoire depuis une dizaine d'années, quelques cas seulement peuvent être rapportés à des envenimations ophidiennes. Les accidents sont imputables aux Naja, Bitis et Causus. Là encore, les signes locaux sonł très spectaculaires : si la piqûre a lieu au boulet, on observe un gonflement important du membre avec lymphangite et des hémorragies, La peau se sphacèle, puis les tissus nécrosés se détachent. La cicatrisation est lente. Dans tous les cas, la mortalité est faible.

Si le gros bétail résiste assez bien, ił n'en est pas de même des poulets qui sont, dans la région de Fort-Lamy, attaqués principalement par des Naja et des Atractaspis : ils tuent les volailles la nuit, au moment où elles ne peuvent se défendre. Dans certains élevages, la présence de Naja nigricollis est une vrale calamité et les pertes sont relativement lourdes. Les moyens habifuels de protection ne donnent qu'une relative sécurité.

\section{CONCLUSIONS}

10 Dans la partie Nord de la République du Tchad, 460 serpents ont été collectés de 1954 à 1965, appartenant à 19 genres et à 24 espèces différentes.

20 Dans la région de Fort-Lamy, les espèces dominantes sont : Psammophis sibilans sibulans (54 p. 100), Cousus rhombeatus (18 p. 100), Atractaspis watsonii (9 p. 100), Naja haje haje (5 p. 100), Naja nigricollis (4 p. 100), Boaedon fuliginosum (4 p. 100) et Bitis ariefans arletans (1 p. 100).

3o Toujours dans la même zone, de Novembre à mai, les serpents sont relativement rares, sauf Psammophis sibilans qui est présent toute l'année. Les Noja, les Causus et les Atractaspis sont abondants, surtout durant l'hivernage ef en arrière-saison (octobre).

4o Des sondages effectués en 1956-1957 autour du Laboratoire de Farcha permettent de fixer le taux d'envenimation ophidienne chez l'homme à environ 0,75 p. 100.

Chez les ruminants, la mortalité par Causus, Naja et Atractaspis semble faible. Par contre, les poulets payent un assez lourd tribut aux morsures des Naja et des Atractaspis.
Institut d'Elevage ef de Médecine vétérinare des pays tropicaux
Laboratoire de Farcha Fort-Lamy (TChad).




\section{SUMMARY}

Notes on the snakes of Chad. Prelminary study of some snakes collected in the Fort Lamy Region from 1954 to 1965

460 snakes, belonging to 19 genera and 24 various species, were collecled from 1954 to 1965 in the Northern part of the Chad Republic.

The survey was mostly carried out in the area near the laboratory of Farcha (Fort Lamy) where the following species were prevalent: Psammophis sibilans sibilans (54 p. 100), Cousus rhombaatus (16 p. 100), Atractospis wotsonu (9 p. 100), Noja haje hoje ( 5 p. 100), Na, a nigricolits (4 p. 100), Booedon fuliginosum (4. p. 100), Bitis arietans arietons (1 p. 100).

In th!s area, the snakes were rare from November to May. They were much more numerous during and just after (October) the rainy season. In human beings, the percentaje of the cases of venimous snake bites recorded in 1956 1957 was about 0,75 p. 100 .

In animals, snake bites result relatively in few death, in chickens.

\section{RESUMEN}

Notas sabre la serpientes de Chad. Estudio preliminar de algunas serpientes recogidas en la regiónde Fort-Lamy de 1954 a 1965

En la parte del norte de la Républica de Chad, se recogieron, de 1954 a 1965, 460 serpientes perteneciendo a 19 géneros y 24 especies diferentes EI autar estudia particularmente la región cerca del Laboratorio de Farcha (FortLamy) donde se encuentran sobre todo las especies siguientes : Psammophis sibilans sibilans ( 54 por 100), Cousus rhombealus (18 por 100), Atractaspis wastsonil (9 por 100), Noja haje hoje (5 por 100), Naja mgricollis (4 por 100), Boaedon fuliginosum (4 por 100) y Bris arlefans oritons (1 por 100) En esic zona, se encuentran pocas serpientes de noviembre a mayo. Son mucho más numerosas durante la estación de las lluvias y al fin del oloño (Octubre).

En el nombre, el porcentaje de los envenenamientos por las serpientes llega a unos 0,75 por 100 en 1956-1957. En los animales, las picaduras de las serpientes causan bastante pocas muertes excepto en los pollitos.

\section{BIBLIOGRAPHIE}

ANGEL (F.). - Les serpents de l'Afrique occldentale françaıse. Bull. Com. Etud. Hist. Scı. A. O. F. 1932 (1933), 15, 613-858, 83 fig.

ANGEL (F.). - Reptiles et amphıbiens du Sahara central et du Soudan. Bull. Com. Etud. Hist. SCI. A. O. F. 1938 (1939), 21, 345-384, 1 tabl.

CONDAMIN (M.). - La collection de serpents de I'I. F. A. N. (acquisition 1956). Bull. l. F. A. N. (1958), 29, ser A., I, 243-262.

CONDAMIN (M.). - Serpents récoltés à Sérédou (Guinée) par R. Pujol. Bull. I. F. A. N. (1959), 21, Ser A., 4, 1350-1366.

CONDAMIN (M.) et VILLIERS (A.). - Contribution à l'étude de la faune de la basse Casamance II. Reptiles. Bull. I. F. A. N. (1962), 24, Sér. A., 3, 897-908.
DEKEYSER (P. L.) et DERIVOT (J.). —A propos des crochets venimeux des Atractaspis. Bull. 1. F. A. N. (1960), 22, Sér. A., 3, 1109-1113.

DOMERGUE (C. A.). - Lisie des Ophidiens de Tunisie. Arch. Inst. Post. Tunis (1959), 36, 2, 157-161.

DOMERGUE (C. A.). - Clé de détermination des serpents de Tunısie et d'Afrique du Nord. Arch. Inst. Past. Tunis (1959), 36, 2, 162-172, 22 clich.

PASTEUR (G.) ef BONS (J.). - Catalogue des reptiles actuels du Maroc. Inst. Sci. Cherif. Ser. Zool. (1960), 21, 132 pp., 5 pl.

PELLEGRIN (J.).- - Reptiles et Batraciens recueillis par la mission Tilho (Niger-Tchad). Doc. Scl. Mis. Titho (1906-1909) (1914), Paris Larose, III, 121-129. 
PHISALIX (M.). - Animaux venimeux et venins (1922), Paris Masson, 864 pp.

ROUSSEL (M. R.) et VILLIERS (A.). - Serpents du Mayo-Kebbi (Tchad). Bull. I. F. A. N. (1965), 27, Sér. A., 4, 1522-1533.

ROUX-ESTÉVE (R.). - Sur une collection de serpents du Nord-Cameroun rapportée par Mr A. Stauch. Bull. Mus. Hist. Not. (1962), $34,2,144-148$.

ROUX-ESTEVE (R.) et GUIBE (J.). - Etude comparée de Boaedon flliginosus (Bolé) et de B. lineatus D. et B. (Ophidieens). Bull. I. F. A. N. (1965), 27, Sér. A., 1, 397-409.
VILLIERS (A.) - Les serpents de l'Ouest africain. Init. afr. I. F. A. N. (1950), 148 pp., 190 fig.

VILLIERS (A.). - Les collections de serpents de I'I. F. A. N. (Dakar) : 1951 : acquisitions 1950. Bull. I. F. A. N., 13, 3, 813-836, 1 fig. ; 1952 : acquisitions 1951. Bull. I. F. A. N., 14, 3, 881-898; 1953 : acquisitions 1952. Bull. I. F. A. N., 15, 3, 1103-1127, 5 fig.

VILLIERS (A.). - Les collections de serpents de I'I. F. A. N. (Dakar) : 1954 : acquisitions 1953. Bull. I. F. A. N., 16, 4, 1234-1247; 1956 : acquisitions 1954-1955. Bull. I. F. A. N. $17,3,877-883$. 